\title{
El quinto pilar de la protección social: la política de dependencia en Francia
}

\author{
Social Protection as the Fifth Estate: Dependency Policy in France
}

\author{
Eguzki Urteaga \\ Universidad del País Vasco \\ eguzki.urteaga@ehu.es
}

Recibido: 09/06/2010

Revisado: 14/07/2011

Aceptado: 28/11/2011

Disponible on line: 15/02/2012

\section{Resumen}

Este artículo analiza la política de atención de la dependencia en Francia. Si las reformas llevadas a cabo durante los últimos años constituyen la base de la política de atención de la dependencia, ésta es todavía insuficiente y no permitirá hacer frente al aumento de las necesidades. Si las últimas reformas han desarrollado y estructurado la política de atención de la pérdida de autonomía, ésta tiene ciertas carencias y deberá enfrentarse a un aumento estructural de las necesidades sociosanitarias. En este sentido, la creación de un quinto riesgo ofrece la oportunidad de realizar unas elecciones estructurantes en términos de socialización del gasto y de reunir las condiciones de una evolución adecuada y eficiente de la oferta de servicios a las personas concernidas.

Palabras clave: política social, dependencia, reforma, Francia.

\begin{abstract}
This article analyzes dependency policies in France. If the reforms carried out during the 2000s constitute the base of the policies of attention for dependency, they are still insufficient and will not allow facing an increase in needs. If a loss of autonomy has been developed and structured by the most recent reform of policies of attention, it has certain gaps and it will have to face a structural increase of social health needs. In this sense, the creation of a fifth risk offers the opportunity to realize a few structuring choices in terms of socialization of spending and of assembling the conditions for a suitable and efficient evolution in the offering of services to those concerned.
\end{abstract}

Keywords: Social policy, Dependency, Reform, France.

Referencia normalizada: Urteaga, E. (2012): «El quinto pilar de la protección social: la política de dependencia en Francia». Cuadernos de Trabajo Social, 25(1): 221-235.

Sumario: Introducción. 1. Las reformas posteriores al año 2000. 2. La creación del quinto riesgo. 3. Conclusiones. 4. Referencias bibliográficas.

\section{Introducción}

La dependencia, que puede definirse como la incapacidad para realizar solo los actos de la vida cotidiana, ha aparecido recientemente como categoría específica de la intervención pública. No formaba parte de los riesgos identificados en el momento de la creación de la Seguridad Social en 1945 (Urteaga, 2009b). La esperanza de vida, de alrededor de 65 años, era mucho menor y se ignoraba ampliamente esta problemática. El problema fundamental de las personas mayores era el de la pobreza y la prioridad era concederles una renta de sustitución. Por lo tanto, los primeros dispositivos que pretendían hacer frente a la pérdida de autonomía se desarrollan fuera de las prestaciones legales: los regímenes de jubilación han intervenido en el marco de la acción social discrecional, lo que ha supuesto un avance aunque no aporte ninguna garantía a las personas. Existían igualmente unas prestaciones legales de ayuda social, pero se dirigían a las 
personas con ingresos reducidos y estaban condicionadas.

En los años noventa, el número de personas dependientes se ha incrementado de manera notable, por lo que se ha buscado una respuesta global. La expresión «quinto riesgo» aparece entonces, traduciendo inicialmente la idea de que el tratamiento de la dependencia no estaba vinculado a ninguno de los cuatro riesgos de la Seguridad Social (familia, enfermedad, vejez y accidentes laborales). Requiere efectivamente unas intervenciones que no son ni del orden del tratamiento médico, que está asumido por el seguro de enfermedad, ni del orden de la garantía de ingresos, asegurada por el sistema de pensiones. Estas intervenciones aspiran no tanto a tratar la enfermedad como a ayudar a la persona a vivir con ella. Pueden consistir en una ayuda humana, que facilita la realización de gestos cotidianos, un dispositivo técnico o unas obras de adecuación de la vivienda.

La referencia a un «quinto riesgo» implica, además, un cierto enfoque político del problema en ruptura con las lógicas discrecionales y restrictivas de los dispositivos anteriores. A diferencia de la acción social, un riesgo de protección social debe fundamentarse en una lógica objetiva: a partir del momento en que la persona cumple las condiciones exigidas, dispone de un derecho subjetivo, que no es tributario de una acción discrecional de la administración pública. A diferencia de la ayuda social, un riesgo asegura una atención universal que no está dirigida exclusivamente a las personas con escasos recursos. En la mente de sus promotores, el quinto riesgo implica igualmente dejar de valorar las necesidades de una persona en función de su edad, sino según su nivel de pérdida de autonomía, lo que exige unificar las políticas destinadas a las personas mayores y discapacitadas (Urteaga, 2010b). En 1962, una comisión presidida por Pierre Laroque, el fundador de la Seguridad Social, afirma lo siguiente:

De cara a la acción social que debe beneficiar a los mayores, la edad cronológica no constituye un criterio válido, sino más bien el nivel de validez, el estado psicológico, la aptitud o la inaptitud a llevar a cabo una vida relativamente independiente» (Laroque, 1962).

Según la Comisión, si la edad podía condicionar la concesión de una renta de sustitución, no podía determinar la atribución de prestaciones que tienen otro objeto, el de paliar la disminución que padecen los discapacitados.

Desde la elección presidencial de 2007, la creación del quinto riesgo es un compromiso oficial del gobierno. No se trata de una creación ex nihilo, dado que ha sido precedida por varias etapas. Dos prestaciones, la ayuda personalizada de autonomía (APA) ${ }^{1}$, creada en 2001, y la prestación de compensación de la discapacidad (PCD) ${ }^{2}$, creada en 2005, han ido en ese sentido. Aunque consideradas formalmente como prestaciones de ayuda social, pueden ser concedidas a cualquier persona sin condición de recursos y sin recurrir a la sucesión. Además, la atención de la dependencia

${ }^{1}$ La ayuda personalizada de autonomía está destinada a las personas mayores que viven en su domicilio, en su familia o en otra casa, o a las personas acogidas en una residencia de ancianos, y que tienen dificultades para realizar las acciones más básicas y sencillas de la vida cotidiana: levantarse y desplazarse, vestirse y salir de casa, preparar la comida y limpiar su hogar. La ayuda personalizada de autonomía tiene como objetivo prioritario facilitar la vida diaria de estas personas tanto en sus hogares como en las residencias en las que se encuentran. En su domicilio, la APA financia varios servicios: personal de ayuda a domicilio, acogida de día, acogida temporal, ayudas técnicas, reforma de la vivienda, transportes, etc. En residencia, la ayuda personalizada de autonomía contribuye a financiar los gastos vinculados a la atención de la dependencia. La residencia puede igualmente concentrar los recursos humanos y materiales sobre esta persona con el fin de ayudarla en su vida diaria.

${ }^{2}$ La prestación de compensación de la discapacidad (PCD) es una ayuda personalizada destinada a financiar las necesidades vinculadas a la pérdida de autonomía de las personas discapacitadas. Estas necesidades deben figurar en un plan personalizado definido por el equipo multidisciplinar de la Casa departamental de las personas discapacitadas (CDPD), sobre la base del proyecto de vida expresado por la persona concernida. Es posible beneficiarse de esta prestación a domicilio o en centro. Esta prestación cubre las ayudas humanas y materiales. 
fue dotada en 2004 de recursos, vinculados a la creación de una «jornada de solidaridad» ${ }^{3} \mathrm{y}$ a un organismo nacional encargado de utilizar estos recursos para el provecho exclusivo de las personas concernidas a través de la Caja nacional de solidaridad para la autonomía $(\mathrm{CNSA})^{4}$.

No en vano, la fase siguiente ofrece la oportunidad de aportar unas respuestas a largo plazo a unos problemas importantes:

- Actualmente, un gran número de hogares hace frente a unas cargas elevadas como consecuencia de la dependencia de uno de sus miembros.

- La oferta de servicios destinados a las personas mayores dependientes, a domicilio o en residencia, sigue siendo insuficiente en cantidad y en calidad.

- El número de personas mayores dependientes aumentará alrededor del 50 por ciento de ahora a 2040.

- Las diferencias entre los dispositivos destinados a las personas mayores y a las personas con discapacidad, sabiendo que estas últimas son a menudo más generosas, dificultan la convergencia.
Para ello, la reforma deberá delimitar el perímetro del quinto riesgo, organizar su gobernanza y, sobre todo, determinar el nivel de socialización del gasto, es decir el nivel de atención prestada por la comunidad, fijando el nivel de atención por las administraciones públicas y las modalidades de toma en consideración de la renta y del patrimonio de las familias para el cálculo de las prestaciones que deben percibir. Inicialmente anunciada para 2008, ha sido pospuesta en varias ocasiones, lo que da cuenta de la dificultad de esta cuestión.

\section{Las reformas posteriores al año $\mathbf{2 0 0 0}$}

Si las reformas llevadas a cabo desde el año 2000 han constituido los fundamentos de la política de atención de la dependencia, ésta sigue siendo insuficiente y no permitirá, en su configuración actual, hacer frente al incremento de las necesidades.

\subsection{El desarrollo y la estructuración de la política de atención de la dependencia}

Las reformas elaboradas en los años 2000 han desarrollado y estructurado la política de atención de la pérdida de autonomía.

${ }^{3}$ La ley del 16 de abril de 2008 relativa a la jornada de solidaridad precisa las modalidades de dicha jornada. Esta consiste en una jornada de trabajo adicional (que puede ser fraccionada en horas) destinada a financiar acciones de solidaridad a favor de la autonomía de las personas mayores o discapacitadas. Esta jornada no da lugar en principio a una remuneración adicional, aunque unos dispositivos estén previstos para los trabajadores que cambian de empleador a lo largo del año para que no tengan que realizar varias jornadas de solidaridad durante el mismo año. Para los trabajadores, esta jornada no constituye una modificación del contrato de trabajo, mientras que para los empleadores, se traduce por una nueva contribución que deben asumir (la «contribución solidaridad autonomía»), ya que el producto de ese día adicional está destinado a financiar unas acciones a favor de la autonomía de las personas mayores y discapacitadas.

La jornada de solidaridad concierne a todos los trabajadores que disponen de un contrato de trabajo. No en vano, en lo que se refiere a los trabajadores de menos de 18 años, conviene tomar en consideración unas disposiciones particulares que rigen el trabajo de los menores. Así, si la jornada de solidaridad está fijada un día festivo, no concernirá a los jóvenes trabajadores, puesto que, excepto en raras ocasiones, el trabajo de los asalariados de menos de 18 años está prohibido durante los días festivos. Si un acuerdo colectivo fija un día no festivo como jornada de solidaridad, los agentes sociales deben posicionarse sobre las condiciones en las cuales estos jóvenes realizarán esta jornada.

${ }^{4}$ La Caja nacional de solidaridad para la autonomía (CNSA) es una entidad pública creada por la ley del 30 de junio de 2004. La ley sobre la igualdad de derechos y oportunidades de las personas discapacitadas del 11 de febrero de 2005 ha precisado y reforzado sus misiones. Puesta en marcha en mayo de 2005, la CNSA tiene la responsabilidad de: 1) financiar las ayudas a favor de las personas mayores dependientes y discapacitadas, 2) garantizar la igualdad de trato sobre todo el territorio y para el conjunto de los discapacitados, 3) y asegurar una misión de asesoramiento, de información y de animación para asegurar el seguimiento del servicio prestado a las personas. Por lo tanto, la CNSA es a la vez una caja encargada de repartir los recursos financieros y una agencia de asesoramiento técnico. 


\subsubsection{Los primeros dispositivos}

El primer dispositivo que ha permitido responder a las solicitudes vinculadas a la dependencia ha sido la ayuda compensatoria para terceras personas $(\mathrm{ACTP})^{5}$, prestación de ayuda social creada por la ley del 30 de junio de 1975; creada al mismo tiempo que la ayuda para adultos discapacitados (AAD) ${ }^{6}$. Aunque fuera destinado inicialmente a los adultos discapacitados, la ausencia de límite de edad de la ayuda compensatoria para terceras personas ha permitido a las personas mayores dependientes acceder a ella. No obstante, la ayuda compensatoria para terceras personas pecaba por una falta de instrumentos de evaluación de la pérdida de autonomía y por su carácter concertado, características que no permiten adaptarla a las necesidades personales (Urteaga, 2010b).

Después de varios intentos infructuosos, la ley del 24 de enero de 1997 crea la primera prestación dedicada a la dependencia de las personas mayores: la prestación específica dependencia ${ }^{7}$. Esta ayuda solucionaba algunos de los defectos de la ayuda compensatoria para terceras personas, dado que se fundamentaba en un instrumento personalizado de la pérdida de autonomía, el baremo $\mathrm{AGGIR}^{8}$ y su cuantía estaba definida en función de esta evaluación. Conservaba, sin embargo, las principales características restrictivas de las prestaciones de ayuda social.

Estas características han conducido a una escasa utilización de estos recursos por las personas que podían beneficiarse de ellas. Mientras que, según el INSEE ${ }^{9}, 800.000$ personas estaban afectadas por una dependencia media o severa, solamente 135.000 gozaban de la prestación específica dependencia en el año 2000. Esta prestación no era la única prestación de ayuda social que beneficiaba a las personas mayores dependientes. Estas accedían también, con las mismas restricciones y unas condiciones de recursos todavía más estrictas, a la ayuda social al alojamiento (ASA) ${ }^{10}$ que permitía a las personas desprovistas estar acogidas en residencias de ancianos, y la ayuda social a domicilio, que financiaba un número limitado de horas de ayuda doméstica, o el reparto de comidas. Las personas mayores podían igualmente beneficiarse de prestaciones extralegales, es decir que dependían de una acción social pública discrecional y no del derecho, financiadas por los regímenes de seguro de vejez. Estas prestaciones podían alcanzar

\footnotetext{
${ }^{5}$ La ayuda compensatoria para une tercera persona (ACTP) es sustituida por la prestación de compensación a partir del 1 de enero de 2006. No obstante, las personas que se benefician de la ACTP antes de esa fecha podrán continuar a percibirla, mientras cumplan las condiciones de concesión y expresen su deseo de continuar a percibirla en cada renovación de sus derechos. Este derecho optativo supone, como contrapartida, una información previa de la persona beneficiaria sobre las cuantías respectivas de ayuda y las prestaciones a los que puede acceder.

${ }^{6}$ La Ayuda para Adultos Discapacitados (AAD) es una prestación que tiene como objeto garantizar una renta mínima a las personas discapacitadas para que puedan enfrentarse a los gastos de la vida corriente. Su pago es subsidiario, puesto que el derecho a beneficiarse de la ayuda es efectivo únicamente cuando la persona discapacitada no puede aspirar a una prestación por vejez, invalidez o a una renta de accidente laboral de una cuantía al menos igual al de la AAD. Esta es concedida a partir de un cierto nivel de discapacidad, siempre y cuando cumpla unas condiciones de residencia y de nacionalidad, de edad y de recursos.

${ }^{7}$ La prestación específica dependencia es una ayuda destinada a pagar los gastos vinculados a la dependencia de las personas mayores de 60 años. Es una prestación en naturaleza, es decir directamente destinada a la financiación de los gastos de ayuda previamente definidos y que se distinguen de la atención médica. Es concedida por el Departamento en nombre de la ayuda social.

${ }^{8}$ El baremo AGGIR (Autonomía Gerontología Grupos Iso-Recursos) constituye un instrumento destinado a valorar el grado de pérdida de autonomía o el grado de dependencia, física y psíquica, de los solicitantes de la ayuda personalizada de autonomía (APA), en la realización de sus actividades diarias.

${ }^{9}$ Instituto Nacional de la Estadística y de los Estudios Económicos.

${ }^{10}$ Cuando las rentas de la persona mayor y la ayuda financiera que sus hijos son susceptibles de concederle en el marco de la obligación alimenticia no le permiten cubrir la totalidad de sus gastos de alojamiento en una residencia de ancianos, la persona mayor puede solicitar la ayuda social de alojamiento para personas mayores. Esta ayuda es concedida por los servicios del Consejo general del Departamento en el cual la persona ha residido durante los tres meses que preceden su ingreso en una residencia de ancianos.
} 
una cuantía significativa y completaban la prestación específica dependencia y las prestaciones de ayuda social legal, atendiendo a las personas cuyo nivel de dependencia era más débil y cuyos recursos eran más elevados. No en vano, tenían los defectos inherentes a la acción social.

\subsection{La creación de la ayuda personalizada de autonomía en 2001}

Creada por la ley del 20 de julio de 2001, la ayuda personalizada de autonomía ha sido concebida como una respuesta al fracaso de la prestación específica dependencia, tomando el traspié de sus aspectos restrictivos y fuentes de desigualdad (Sueur, 2000). Si bien la ayuda personalizada de autonomía tiene varias características de la prestación específica dependencia, se diferencia de ella por los siguientes aspectos:

- se trata de una prestación universal, no sometida a condición de recursos, ya que cualquier persona que depende de los GIR ${ }^{11}$ tiene derecho a la ayuda personalizada de autonomía;

- no es subsidiaria con respecto a los créditos alimenticios;

- no está sometida al impuesto de sucesiones;

- su baremo es idéntico sobre el conjunto del territorio.

La ayuda personalizada de autonomía es una prestación en naturaleza: a diferencia de una pensión de jubilación, no tiene como objeto aportar una renta a su beneficiario, sino financiar unos gastos causados por la pérdida de autonomía. La ayuda personalizada de autonomía puede ser concedida a una persona que vive en su domicilio y en una residencia para per- sonas mayores. Cuando es concedida a domicilio, financia las ayudas, especialmente las horas de intervención de una persona asistente, previstas por un plan de ayuda establecido por el equipo médico-social. Puede financiar incluso la ayuda de un miembro de la familia o de un ayudante familiar, que puede ser remunerado hasta el 85 por ciento del salario mínimo $(\mathrm{SMIC})^{12}$. Cuando es concedida a las personas en residencia, financia la tarifa de dependencia de la residencia, es decir la parte del coste soportado por una residencia que se refiere a la atención de la dependencia de los residentes.

La cuantía de la ayuda personalizada de autonomía está calculada en función del nivel de dependencia medido por el grupo iso-recursos (GIR) y del nivel de recursos. Existe para cada grupo una cuantía máxima. Cuando la persona tiene unos recursos inferiores a 689.50 euros (baremo de 2009), recibe la cuantía máxima. Cuando sus recursos son superiores, debe pagar un «ticket moderador» que se incrementa con la renta.

La creación de la ayuda personalizada de autonomía ha dado lugar a unos debates sobre su gobernanza y financiación. Dado que la ayuda social ha sido ampliamente descentralizada durante los años 1980, las prestaciones legales que asumen los costes inherentes a la dependencia, entre las cuales figura la prestación específica dependencia, dependían de los Departamentos cuyos esfuerzos eran variables (Urteaga, 2010a). En la lógica del paso a una prestación universal y homogénea sobre el conjunto del territorio, habría supuesto confiar su gestión y financiación a los regímenes de vejez de la Seguridad social. No en vano, por pragmatismo, el gobierno ha preferido mantener la competencia en los Departamentos, que disponían de los recursos humanos necesarios para

${ }^{11}$ Los grupos iso-recursos (GIR) permiten clasificar a las personas en función de las diferentes etapas de pérdida de su autonomía. Son un total de seis. La clasificación en un GIR se realiza en función de los datos recaudados por un equipo médico-social gracias al baremo AGGIR que permite ponderar diferentes variables (coherencia, orientación, limpieza, comunicación, etc.).

${ }^{12}$ El salario mínimo interprofesional de crecimiento (SMIC), antiguamente salario mínimo interprofesional garantizado (SMIG), es el salario mínimo horario por debajo del cual ningún trabajador puede ser remunerado. Es revalorizado anualmente el 1 de enero. A diferencia del SMIG basado en la inflación, el SMIC es revalorizado como mínimo a la mitad del aumento del poder adquisitivo del salario horario de base obrera $(\mathrm{SHBO})$ y puede beneficiarse de un incremento adicional bajo decisión gubernamental. Al 1 de enero de 2010, el salario mínimo se sitúa en 1.348,80 euros mensuales brutos, lo que corresponde a $1.055,42$ euros mensuales netos. 
evaluar la dependencia. En otros términos, ha decidido poner en marcha un «quinto riesgo», a través de una atención objetiva y universal de la dependencia, pero no un «quinto sector», lo que habría implicado una organización institucional que dependiera de la Seguridad social.

La ley del 10 de julio de 2001 crea, por primera vez, una prestación descentralizada cuyo baremo está totalmente definido a nivel nacional. Otros casos han seguido, con la descentralización de la renta mínima de inserción ${ }^{13}$ en 2003, la creación de la prestación compensatoria de la discapacidad en 2005 o la de la renta de solidaridad activa (RSA) ${ }^{14}$ en 2008 (Urteaga, 2011). Esta arquitectura presenta unas dificultades para los Consejos generales, ya que estos últimos deben soportar una fuerte carga, cuyos determinantes se les escapan ampliamente y sobre la cual tienen escasos márgenes de maniobra. Si algunos consideran que asegura un buen equilibrio, entre igualdad de derechos sobre el territorio nacional y gestión de proximidad, otros la critican porque consideran que se trata de una «descentralización de taquilla» (Mercier, 2005).

Al contrario de la prestación específica dependencia, el número de beneficiarios de la ayuda personalizada de autonomía supera rápidamente las previsiones iniciales. Mientras que el estudio de impacto que acompañaba el proyecto de ley contemplaba 550.000 beneficiarios al término de la fase de desarrollo, este número ha sido superado a partir del primer año de entrada en vigor y no ha cesado de progresar desde entonces a un ritmo rápido alcanzando 1.115.000 personas el 31 de diciembre de 2008 .

\subsubsection{El desarrollo planificado de la oferta de servicios}

La política de atención de la dependencia no se reduce a unas prestaciones sociales que financian las cargas soportadas por las personas. Implica el desarrollo de estructuras de atención adaptadas cuantitativamente (número de plazas) y cualitativamente (adaptación a las características del público y especialmente al nivel de dependencia, calidad y profesionalismo del servicio). Estas estructuras están ampliamente financiadas por las administraciones públicas, en parte por las prestaciones de ayuda social, entre las cuales se encuentra la ayuda personalizada de autonomía, y parcialmente por el seguro de enfermedad. Existen dos categorías de estructuras: las estructuras de alojamiento y las estructuras de ayuda a domicilio.

Francia contaba en 2003 con 671.000 plazas de alojamiento para personas mayores, es decir 140 plazas para 1000 personas de más de 75 años. Esta tasa de equipamiento es relativamente elevada comparándola con el de sus vecinos europeos. Se observa de manera continua un retroceso de la edad de ingreso en residencias y un aumento del gasto medio de los residentes. El ingreso en residencia se produce cada vez más durante los últimos años de la vida, cuando el nivel de dependencia se convierte en demasiado elevado para permitir el mantenimiento a domicilio.

La oferta de servicios a domicilio está mucho menos desarrollada. Se contabilizaban en 2004 solamente 82.000 plazas de servicios de cuidados enfermeros a domicilio (SSIAD) ${ }^{15}$, es decir menos de 17 plazas para 1000 habitantes

${ }^{13}$ La Renta Mínima de Inserción es una prestación en vigor desde el 1 de diciembre de 1988 y hasta el 31 de mayo de 2009. Financiada por el Estado y gestionada por los departamentos, es concedida por las Cajas de ayuda familiar (CAF) o la Mutua social agrícola (MSA) a las personas sin recursos o que tienen ingresos inferiores al umbral fijado por decreto. La renta mínima de inserción ha sido sustituida en 2009 por la Renta de solidaridad activa.

${ }^{14}$ La renta de solidaridad activa es una renta mínima entrada en vigor el 1 de junio de 2009. Se trata de una prestación concedida a las personas que trabajan pero cuyos recursos financieros son limitados. Su cuantía depende a la vez de la situación familiar y de las rentas del trabajo. El Estado y los Departamentos se han asociado para poner en marcha esta nueva prestación. La renta de solidaridad activa en concedida por las Cajas de ayuda familiar o las Cajas de mutualidad social agrícola, y concierne a 3 millones de hogares. Sustituye la renta mínima de inserción, la ayuda padre aislado (API) y ciertas ayudas tales como la prima de retorno al empleo.

${ }^{15}$ Los Servicios de Atención Enfermera a Domicilio constituyen un elemento fundamental de la atención enfermera a domicilio de las personas mayores y muy mayores, enfermas y a menudo dependientes para la realización de los actos básicos de la vida cotidiana. Estos servicios tienen como objetivo mantener a la persona mayor o dependiente en su entorno vital en las mejores condiciones posibles, de prevenir y de 
de más de 75 años, y 54.000 enfermeras autónomas. No obstante, el 88 por ciento de las personas mayores de más de 75 años y todavía el 75 por ciento de los más de 85 años viven a domicilio, y un gran número de ellas necesita una ayuda. Por lo tanto, esta ayuda está asegurada en gran medida por la familia.

La canícula del verano de 2003 cuestiona gravemente la adecuación de esta oferta de servicios a las personas mayores dependientes. $\mathrm{Si}$ se valora en 15.000 el número total de fallecimientos causados por la canícula, la tasa de mortalidad ha sido muy superior en las residencias de ancianos $(+100$ por ciento) comparándola con la observada tanto en los hospitales $(+50$ por ciento) como a domicilio $(+70$ por ciento). Si esta mortalidad especialmente elevada se explica en parte por la gran vulnerabilidad de las personas acogidas en residencias de ancianos, las investigaciones han puesto de manifiesto las insuficiencias del parque de alojamiento en lo que se refiere a la adaptación de los locales, la formación de los profesionales y la medicalización.

Dos planes plurianuales de fortalecimiento de la oferta de servicios en residencia y a domicilio son elaborados posteriormente a la canícula: el plan «Envejecimiento y solidaridades» para el periodo 2004-2007 y el plan «Solidaridad edad avanzada» para el periodo 2007-2012. El primero contemplaba la creación de 17.000 plazas de SSIAD, 10.000 plazas de alojamiento y 15.000 plazas de enfermeras $\mathrm{y}$ auxiliares en las residencias de ancianos. El segundo, mucho más ambicioso, programaba la creación durante ese mismo periodo de 30.000 plazas de alojamiento y de 40.5000 plazas de SSIAD.

Existe una diferencia notable entre los enfoques privilegiados por el sector del alojamiento y el de los servicios a domicilio. En materia de alojamiento, es cuestión sobre todo de promover una recuperación cualitativa, para adaptar las estructuras al nivel más elevado de dependencia de sus residentes. Esta recupera- ción consiste en aumentar el número de estructuras medicalizadas, proponiendo unos servicios de cuidado gracias a la presencia de un médico coordinador, así como mejorar la tasa de atención personal, es decir el número de personas que prestan los servicios a las personas mayores con respecto al número de residentes. La política de medicalización de las estructuras fue iniciada por la ley 1997 que creó la prestación específica dependencia. Después de varios años de lenta progresión durante los cuales el objetivo del 100 por ciento de residencias de ancianos medicalizadas ha sido aplazado, los planes plurianuales han permitido aproximarse a ello de manera sustancial: mientras que la tasa de plazas medicalizadas solo era del 31 por ciento en 2003, pasó al 75 por ciento en $2007^{16}$. En términos cuantitativos, si los planes han permitido proseguir el aumento del número de plazas en residencias en valor absoluto, estas han progresado menos rápidamente que la población de personas mayores, y la tendencia a la bajada de la tasa de equipamiento no se ha interrumpido.

No obstante, en materia de ayuda a domicilio, se busca una verdadera recuperación cuantitativa, que prolonga una tendencia anterior, dado que el número de plazas en SSIAD se vio triplicado entre 1986 y 2004. Debería alcanzar 140.000 en 2012, es decir una casi-duplicación en menos de diez años. El objetivo perseguido es promover la «libre elección», facilitando en la medida de lo posible el mantenimiento a domicilio. Es la política elegida por un gran número de países de la OCDE bajo la denominación «desinstitucionalización».

\subsubsection{El gasto dedicado a la dependencia}

Una misión de información realizó en 2008 una evaluación del conjunto del gasto dedicado a la dependencia (Vasselle, 2008). Se distinguían dos categorías: los gastos directamente afectados a la dependencia, que alcanzan cerca de 16.000.000.000 euros y los gastos de cuidados realizados tanto por los

retrasar las hospitalizaciones, de reducir la duración de las estancias en centros hospitalarios, de favorecer y organizar de manera harmoniosa la vuelta al domicilio. Para beneficiarse de dichos servicios, es suficiente ser una persona mayor de 60 años y gozar de la cobertura de la Caja de enfermedad o de ser una persona de menos de 60 años que padece enfermedades incurables o que generan discapacidad, sobre la base del informe del médico de la Caja primaria de seguro de enfermedad.

${ }^{16}$ DRESS, Enquêtes EHPA, Etudes et résultats, n³79 et 689. 


\begin{tabular}{|l|c|l|}
\hline \multicolumn{1}{|c|}{ Gasto } & Cuantía & \multicolumn{1}{|c|}{ Fuente de financiación } \\
\hline Ayuda personalizada de autonomía & 4,6 & Departamentos y CNSA \\
\hline Ayuda social al alojamiento & 1,2 & Departamentos \\
\hline Centros y servicios sociales y médico-sociales & 7,8 & Seguro de enfermedad y CNSA \\
\hline $\begin{array}{l}\text { Gastos de mantenimiento a domicilio de los regíme- } \\
\text { nes de jubilación }\end{array}$ & 0,3 & Regímenes de jubilación \\
\hline Gastos fiscales del Estado & 0,4 & Estado \\
\hline $\begin{array}{l}\text { Exoneración de cargas sociales para los trabajadores } \\
\text { empleados por unos beneficiarios de la APA }\end{array}$ & 1,5 & Seguro de enfermedad \\
\hline Subtotal & 15,7 & - \\
\hline $\begin{array}{l}\text { Cuidado médico y de hospitalización de las personas } \\
\text { mayores dependientes }\end{array}$ & 4,6 & Seguro de enfermedad \\
\hline Total & 20,3 & - \\
\hline
\end{tabular}

Cuadro 1. Gasto, cuantía y fuente de financiación de la dependencia en 2009.

médicos de cabecera como por los hospitales en favor de las personas mayores dependientes, que están valorados en 4.600.000.000 euros. El cuadro 1 recapitula los principales gastos y muestra la multiplicidad de las fuentes de financiación:

\subsection{Carencias y aumento estructural de las necesidades}

\subsubsection{Un gasto importante para las familias} A pesar de la existencia de la ayuda personalizada de autonomía, las personas que han recurrido a unos servicios de ayuda a domicilio o de alojamiento soportan una parte de su coste. En materia de ayuda a domicilio, la parte restante resulta de la limitación de los planes de ayuda en función del GIR y del mecanismo del «ticket moderador». Este sigue siendo significativo pero limitado, de 114 euros al mes. No obstante, la cuantía restante tiene cierta tendencia a aumentar porque los máximos de los planes de ayuda están revalorizados menos rápidamente que el coste de los servicios a domicilio, que tiende a incrementarse en el mismo ritmo que los salarios.

La suma restante es más importante para las personas que viven en residencias de ancianos. Una misión del IGAS $^{17}$ lo valoraba en 2.200 euros mensuales de media (Branchu et al., 2009). Resulta en parte del «ticket moderador» de la ayuda personalizada de autonomía, pero sobre todo de la parte «alojamiento» de la tarifa de residencia, que no está asumida por la ayuda personalizada de autonomía. La ayuda social al alojamiento y las ayudas personales a la vivienda pueden financiar todo o parte de esta tarifa de alojamiento, pero solo se conceden a las personas con escasos recursos. Dado que el nivel de renta media de las personas residentes es de 1.100 euros al mes, la gran mayoría de las personas residentes deben utilizar su patrimonio o el de sus familiares para financiar su estancia.

${ }^{17}$ La Inspección General de los Asuntos Sociales (IGAS) es el servicio interministerial de control, de auditoría y de evaluación de las políticas sociales para ayudar las administraciones públicas a tomar decisiones. Reúne a 130 expertos de la cohesión social (Familia, Protección de la infancia, lucha contra la exclusión, trabajo social), de la protección social (Seguridad social, prestaciones sociales, etc.), del trabajo, de la formación profesional y de la Sanidad. Cada año elabora entre 220 y 230 informes según unos enfoques que privilegian la calidad y el rigor. 


\subsubsection{Una imagen negativa de la política de atención de la dependencia}

Ese coste elevado contribuye a la imagen negativa del sistema de atención de la dependencia que tienen los ciudadanos franceses. En un sondeo de opinión reciente (Baromètre, 2009), se explicaba a las personas interrogadas que un alojamiento en residencia de ancianos costaba de media 2.200 euros mensuales. El 76 por ciento de las personas encuestadas declaraban que su familia no podría asumir semejante carga financiera. Por último, el 71 por ciento decía que la atención de las personas mayores por los poderes públicos era poco o nada satisfactoria. Es interesante observar que esta percepción se ha deteriorado notablemente estos últimos años, a pesar de los esfuerzos mencionados anteriormente. Así, solamente el 55 por ciento de los franceses consideraban que la atención era insatisfactoria en 2002. Además, la proporción de personas que piensan que no se habla suficientemente de estos problemas en Francia pasó del 63 por ciento en 2003 al 78 por ciento en 2009. Esta percepción resulta de una conciencia creciente de los problemas de la dependencia causados por el envejecimiento de la población: un tercio de los ciudadanos galos declara hoy en día tener en su entorno a una persona mayor dependiente.

Ante una atención residencial muy costosa y percibida como de mala calidad, las personas mayores y sus familias intentan atrasar al máximo el ingreso en una institución. A pesar del notable desarrollo de los servicios a domicilio, sobre todo gracias a su financiación por la ayuda personalizada de autonomía, la ayuda informal de la familia sigue siendo indispensable para asegurar el mantenimiento a domicilio en el 79 por ciento de los casos. Considerado como un «nuevo grupo social» por la SOFRES, los ayudantes familiares son cada vez más numerosos y expresan unas importantes expectativas de apoyo: más del 90 por ciento consideran que es indispensable o útil proporcionar una ayuda financiera, una formación, la posibilidad de tener unos momentos de descanso gracias a unas fórmulas de alojamiento temporales así como un estatus legal.

\subsubsection{Un aumento estructural de las necesi- dades}

La anticipación de las necesidades en materia de atención de la dependencia implica un razonamiento que se divide en tres tiempos: 1) la proyección del número de personas mayores dependientes; 2) la determinación de la oferta de servicios a domicilio y en centros; y 3 ) el cálculo del coste asociado a esta oferta de servicios. Cada una de estas etapas se fundamenta en une serie de hipótesis.

El número de personas mayores de más de 75 años puede ser previsto con un alto nivel de fiabilidad. Una clara inflexión debería producirse en 2020, con la llegada a esa edad de las generaciones del baby-boom: la tasa de crecimiento anual debería pasar del 1 por ciento antes de esta fecha al 3 por ciento después de ella. La proporción de personas mayores dependientes es, por el contrario, mucho más incierta. La dependencia está causada mayoritariamente por cuatro patologías: la artrosis, los accidentes vasculares cerebrales, los problemas coronarios y las demencias, tales como la enfermedad de Alzheimer. Si se ha observado en el pasado un aumento de la esperanza de vida en buena salud, es difícil prever a qué ritmo se producirá, sobre todo para la enfermedad de Alzheimer de la que se desconocen precisamente los determinantes. El INSEE y la DRESS $^{18}$ han realizado unas proyecciones al horizonte 2040, privilegiando dos escenarios. En ambos casos, la progresión del número de personas mayores dependientes debería ralentizarse a partir de 2012, antes de acelerar de nuevo en 2030.

El paso de una proyección en número de personas dependientes a la planificación de una oferta de servicios es de una gran complejidad, lo que explica que ese ejercicio se interrumpa en 2025 y no en 2040. La naturaleza del ejercicio es igualmente diferente de la primera etapa: ya no se trata solamente de prever unas evoluciones ampliamente padecidas por las administraciones públicas, incluso si la política sanitaria tiene cierta influencia sobre la dependencia, sino de planificar unas elecciones. La hipótesis central es la continuación de la desinstitucionalización: si la proporción de personas muy de-

\footnotetext{
${ }^{18}$ Dirección de la Investigación, de los Estudios, de la Evaluación y de las Estadísticas.
} 
pendientes de más de 70 años en residencias debe ser estable en sus dos tercios, la de las personas medianamente dependientes debe bajar del 35 por ciento al 23 por ciento.

Por lo tanto, sería posible estabilizar el número de plazas en residencias en 680.000 a partir de 2010, lo que equivaldría a una disminución rápida de la tasa de equipamiento, teniendo en cuenta el fuerte aumento del número de personas dependientes a lo largo de ese periodo. La mejora cualitativa continuaría, especialmente con un fuerte incremento de la tasa de encuadramiento, que pasaría de 57 equivalentes jornadas completas para 100 residentes a 76 para 100 residentes en un escenario bajo y a 81 para 100 en un escenario alto. Por el contrario, el objetivo de «libre elección» implica proseguir el desarrollo cuantitativo de la oferta a domicilio: así, el número de plazas en SSIAD alcanzaría 229.000 en 2025, es decir el triple de 2004.

La evaluación financiera de las necesidades implica una última serie de hipótesis, especialmente sobre la progresión de los salarios, dado que la parte fundamental del coste de la dependencia está representada por los costes de personal. Suponiendo que los salarios del sector progresarán al mismo ritmo que el salario medio, con una tasa de crecimiento anual de este último del 1,7 por ciento, el gasto dedicado a la dependencia pasaría del 0,94 por ciento del PIB en 2005 al 1,5 por ciento en 2025 .

\section{La creación del quinto riesgo}

La creación de un quinto riesgo es la oportunidad de realizar unas elecciones estructurantes en términos de socialización del gasto y de reunir las condiciones de una evolución adaptada y eficiente de la oferta de servicios a las personas concernidas. Las proyecciones presentadas anteriormente permiten anticipar la evolución del coste de atención de la dependencia. No precisan las modalidades de su financiación, y menos aun del grado de socialización de ese gasto, siendo la principal cuestión que debe zanjar la reforma que instaurará el quinto riesgo con el fin de poner en marcha una gobernanza eficaz.

\subsection{Las elecciones estructurales a realizar}

El informe Gisserot-Grass ha propuesto tres escenarios diferentes según el nivel de socialización de la atención de la dependencia:
- ausencia de incremento de los impuestos, lo que tiene como contrapartida el retroceso de la socialización;

- aumento de los impuestos destinados a financiar un mantenimiento de la socialización y una mejora cualitativa de la oferta de servicios;

- incremento superior aun de la presión fiscal para financiar una mayor socialización.

\subsubsection{Las vías de una mejor cobertura de la cantidad pendiente de pago}

Varias evoluciones pueden ser contempladas para limitar la cuantía pendiente de pago. A domicilio, estos están concentrados sobre las personas severamente dependientes, dado que la limitación de la ayuda no permite siempre financiar el número de horas necesarias. Por lo tanto, sería útil subir el techo para los GIR 1 y 2. Otra propuesta, dirigida en función del nivel de renta en lugar del nivel de dependencia, consiste en transformar, cuando el empleador es una persona mayor dependiente, la reducción del impuesto sobre la renta del 50 por ciento para la contratación de un trabajador a domicilio en crédito de impuesto, lo que abriría su beneficio a la mitad de los hogares noimponibles.

La cantidad de dinero pendiente de pago concierne, sin embargo, las personas acogidas en centros. Una misión de información senatorial (Vasselle, 2008) propone jugar sobre unas transferencias entre las secciones tarifarias de las residencias de ancianos: los gastos computados en la tarifa «cuidados» están totalmente reembolsados por el seguro de enfermedad, las de la tarifa «dependencia» están parcialmente reembolsadas por la ayuda personalizada de autonomía, mientras que las de la tarifa «alojamiento» no están reembolsadas en absoluto, excepto para las familias muy modestas que se benefician de la ayuda social para el alojamiento. Haciendo bascular la totalidad del coste de los auxiliares de la sección dependencia hacia la sección cuidados, la suma pendiente de pago disminuiría de 200 euros mensuales. Otra vía más ambiciosa, que además mejoraría ampliamente la legibilidad del sistema, consistiría en fusionar el conjunto de las prestaciones solventando el alojamiento de las personas mayores dependientes en un único dispositivo y en aumentar su techo. El baremo de este últi- 
mo determinaría claramente el nivel de la suma pendiente de pago en función de las rentas.

Además de su coste, estas medidas plantean dos tipos de dificultades. Por una parte, sería necesario asegurarse de que la mejora de las prestaciones financiadas por las administraciones públicas no esté absorbida por un incremento de los precios exigidos por los prestatarios de servicios, lo que anularía el efecto positivo sobre la suma pendiente de pago. Según la misión senatorial, desde la creación de la ayuda personalizada de autonomía, las personas ingresadas en residencias solo habrían asistido a una reducción de sus cargas de una cuarta parte en comparación con los recursos públicos nuevamente concedidos, dado que el resto estaba absorbido por la inflación. Por otra parte, las diferentes propuestas contempladas no estarían asumidas por los mismos financiadores. La toma en consideración del interés de las personas y el equilibrio global de las finanzas públicas se complica con unos retos complejos en cuanto al reparto de la carga entre los financiadores.

\subsubsection{Las dificultades de convergencia en- tre los dispositivos}

La supresión de los límites administrativos entre los dispositivos de compensación de la pérdida de autonomía de las personas mayores y de las personas discapacitadas se inscribe en una disposición de principio de la ley sobre la discapacidad del 11 de febrero de 2005. Esta implica especialmente poner fin a la dualidad entre la ayuda personalizada de autonomía, reservada a las personas de más de 60 años, y la prestación de compensación de la discapacidad, cuyo beneficio solo puede ser solicitado por debajo de ese límite. Constituye para algunos, especialmente los actores reunidos en el seno del consejo de la Caja nacional de solidaridad para la autonomía (agentes sociales, asociaciones representativas de personas mayores y discapacitadas), una dimensión fundamental de la creación del quinto riesgo.

Si la ayuda personalizada de autonomía y la prestación de compensación de la dependencia $(\mathrm{PCH})$ tienen varias características comunes, es un dispositivo mucho más generoso. No hay, como para la ayuda personalizada de autonomía, ninguna limitación del nivel de la ayuda, ya que este está realmente determinado en fun- ción de las necesidades de compensación, lo que permite alcanzar para los discapacitados severos unas cuantías muy elevadas. El «ticket moderador» es más débil y solo progresa muy lentamente en función de las rentas. Por lo tanto, el alineamiento de la ayuda personalizada de autonomía sobre la prestación de compensación de la dependencia sería muy costoso: 8 millones de euros, es decir una triplicación del coste de la ayuda personalizada de autonomía.

Los principales informes administrativos y parlamentarios publicados desde 2007 sobre el tema tienden, por lo tanto, a recordar las justificaciones de principio que tienden a distinguir ambas políticas. A diferencia de la discapacidad, la dependencia es un riesgo previsible contra el cual es posible asegurarse o constituir un ahorro. Además, la capacidad de las personas discapacitadas para constituir unas rentas a lo largo de su vida profesional es lo más a menudo reducida. Por lo tanto, parecería lógico que la vida política en favor de las personas discapacitadas presente un nivel de solidaridad superior. Esta sensación se encuentra en las encuestas de opinión: el 74 por ciento de los franceses piensan que incumbe principalmente a las administraciones públicas atender a las personas discapacitadas, frente al 56 por ciento en el caso de las personas mayores dependientes (DRESS, 2006).

La convergencia de los dispositivos podría, por lo tanto, reducirse a unas evoluciones técnicas como la unificación de las modalidades de evaluación de la discapacidad y de la dependencia, y la constitución de una taquilla única que agruparía en cada Departamento a las personas discapacitadas y los servicios dedicados a la acogida y a la orientación de las personas mayores dependientes en una Caja departamental de la autonomía. La otra gran obra común es la contratación del personal necesario para la realización de estas misiones.

\subsubsection{La toma en consideración de la renta y del patrimonio}

Excepto si se aumentan notablemente los impuestos, la mejora de la cobertura de las cuantías pendiente de pago solo podrá hacerse de manera uniforme para todas las personas. Por lo tanto, se contemplan varias medidas para incrementar la toma en consideración de la situación financiera en el cálculo de la ayuda personalizada de autonomía. La vía más simple 
consistiría en incrementar el carácter decreciente de la ayuda personalizada de autonomía en función de la renta. La universalidad de la ayuda personalizada de autonomía correría el riesgo, sin embargo, de convertirse en ampliamente ficticia: hoy por hoy, una persona sola que gana más de 2.750 euros mensuales soporta un «ticket moderador» del 90 por ciento.

Otra vía consiste en fundamentarse no tanto en la renta como en el patrimonio de la persona. Las personas cuyo patrimonio es superior a un cierto umbral (200.000 euros, lo que convertiría la medida en aplicable a un tercio de los hogares de más de 70 años) se vería proponer una elección entre el beneficiario de una ayuda personalizada de autonomía disminuida a la mitad o el mantenimiento de una ayuda personalizada de autonomía a tasa plena, pero acompañada de la imposición de una fianza sobre su patrimonio, de una cuantía de 20.000 euros. Esta medida, cuya aplicación sería progresiva, permitiría ahorrar mil millones de euros cada año a partir de 2013.

\subsubsection{Las soluciones contempladas para fi- nanciar las nuevas necesidades}

Consisten, bien en la generación de nuevos ingresos, bien en la realización de medidas de ahorro que posibiliten la implementación de nuevas medidas. Para los partidarios de una estabilidad de la presión fiscal, la generación de nuevas fuentes de financiación debe hacerse por un nuevo despliegue de los diferentes componentes de la protección social. La rama familia de la Seguridad social es estructuralmente excedentaria, porque sus gastos aumentan con la inflación, mientras que sus ingresos progresan al ritmo de la masa salarial, que es superior. Es la razón por la cual la misión de información senatorial ha propuesto la afectación de una parte de estos excedentes, aludiendo a la vinculación de la ayuda a los ayudantes familiares con las finalidades de la política familiar. Sin embargo, el rápido deterioro de los ingresos de la Seguridad social provocado por la crisis ha cuestionado, al menos para algunos años, esta posibilidad, cuyo principio es discutido. Los partidarios de la creación de nuevos ingresos promueven un aumento de la contribución social generalizada $(\mathrm{CSG})^{19}$, especialmente sobre las rentas del capital y los productos de la inversión bursátil. Un aumento del 1 por ciento de la contribución social generalizada sobre estos ingresos supondría un ingreso adicional de 1.500.000.000 euros.

Las medidas de ahorro avanzadas se refieren a unos nuevos despliegues entre las estructuras sanitarias y la regulación de las tarifas practicadas por los centros de alojamiento. 30.000 camas hospitalarias podrían ser reconvertidas en plazas de alojamiento para personas mayores dependientes. Resultaría de todo ello un ahorro neto de mil millones de euros para las finanzas públicas. La creación de las Agencias regionales de salud (ARS) ${ }^{20}$ por la ley «hospital, pacientes, salud y territorios» de 2009, competentes sobre los sectores sanitario y médico-social, debe facilitar estas reestructuraciones. En lo que se refiere a la tarificación, la reforma de 1997 debía conducir a lo que las dotaciones consentidas a los centros de alojamiento solo dependan de la severidad de la dependencia y de la patología de las personas acogidas, lo que debería incentivar los centros a controlar sus costes. En la práctica, las situaciones históricas más favorables de ciertos centros han sido ampliamente preservadas. Es la razón por la cual las leyes de financiación de la Seguridad social para 2008 y 2009 instauran un sistema de tarifas máximas.

\subsection{La organización del quinto riesgo}

La puesta en marcha de una atención eficaz de la dependencia no es solamente una cuestión

${ }^{19}$ La contribución social generalizada (CSG) es un impuesto destinado a diversificar las fuentes de financiación de la Seguridad social. Las rentas sometidas a ese impuesto son aquellas personas residentes en Francia para el establecimiento del impuesto sobre la renta y que cotizan al régimen obligatorio del seguro de enfermedad. En principio, la contribución social generalizada se aplica a los mismos elementos de remuneración que las cotizaciones a la Seguridad social, con algunas excepciones.

${ }^{20}$ La Ley del 21 de julio de 2009 sobre la reforma del hospital y relativa a los pacientes, a la sanidad y a los territorios, crea, en su artículo 118, las Agencias regionales de sanidad que constituyen el pilar de la reforma del sistema sanitario. Las ARS han sido creadas con el fin de asegurar la dirección unificada de la sanidad en las regiones, de mejorar la respuesta a las necesidades y de incrementar la eficacia del sistema. 
de financiación. Implica que los poderes públicos dirijan eficazmente la evolución de las estructuras, de manera a que las plazas previstas sean creadas en unos plazos razonables, que las personas sean contratadas en cantidad y calidad suficientes o que el reparto territorial de los recursos responda a las necesidades. Se trata del principal reto de la definición de las modalidades de gobernanza del sector.

\subsubsection{La necesidad de una dirección con- junta de las estructuras}

El desarrollo durante los años y las décadas posteriores de una oferta de servicios adaptada a las necesidades implica llevar a cabo paralelamente varias transformaciones:

— La creación entre 2003 y 2010 de 50.000 plazas en residencias de ancianos medicalizadas;

- La creación durante el mismo periodo de 30.000 plazas de alojamiento temporal;

- La renovación de entre 150.000 y 480.000 plazas de alojamiento según las estimaciones, con el fin de respetar las normas de calidad y de llevar la oferta a nivel de las expectativas cualitativas de los usuarios;

- La creación entre 2004 y 2005 de 150.000 plazas de SSIAD.

No obstante, estos datos son insuficientes para llevar a cabo estas operaciones y movilizar las financiaciones necesarias. Conviene igualmente disponer de una valoración de las necesidades por territorio para saber dónde crear las plazas, encontrar unos acuerdos entre los prefectos (delegados del gobierno), y sobre todo controlar la eficiencia de los trabajos de creación y de restructuración con el fin de evitar la superación de los presupuestos previstos. El riesgo es real, como lo muestra el incremento de los costes observado para el Plan de solidaridad edad avanzada: valorada en 2006 en 2.300.000.000 euros, su coste ha sido revalorado en 2008 en 4.600.000.000 para el periodo 2008-2012.

\subsubsection{Las dificultades de contratación del personal}

La progresión de la dependencia implica una notable extensión del número de personas que trabajan en la atención de las personas mayores dependientes, cuyo número debería pasar según las
CAS de 608.900 en 2005 a menos de 1.055 .000 en 2025. El sector de la ayuda a domicilio representaría el 80 por ciento de ese incremento. Teniendo en cuenta el número elevado de jubilaciones, la cantidad de contrataciones a realizar sería todavía más elevado: de 350.000 para la atención en residencias para el periodo 2005-2015.

Varias dificultades obstaculizan la realización de este ambicioso programa de contratación. El sector de la atención de las personas mayores dependientes se enfrenta a la competencia de otros sectores que proponen unos oficios próximos y que tienen igualmente unas importantes necesidades, como el cuidado de los niños o los demás servicios a domicilio. Sufre de una imagen relativamente negativa y de la dificultad inherente a unos oficios que consisten en cuidar de las personas mayores, cuyas capacidades son a menudo muy limitadas en los últimos años de su vida. Por último, la necesidad cuantitativa de realizar numerosas contrataciones puede entrar en conflicto con la preocupación por hacer progresar la calificación de los intervinientes, de manera a mejorar la calidad de la oferta de servicios.

A pesar de estas dificultades, esta fuente de empleos representa una gran oportunidad para un país como Francia, donde la tasa de desempleo sigue siendo elevada. Los primeros niveles de estos empleos son accesibles a unas personas poco cualificadas y pueden jugar un rol de inserción importante. Desde 2008, el Estado intenta federar las iniciativas de formación y de promoción del sector en unos planes profesionales comunes a los sectores de las personas mayores, de las personas discapacitadas y de la pequeña infancia. La parte fundamental de los medios depende sin embargo de las Regiones, que son las únicas responsables desde la descentralización de 2004 de las formaciones sanitarias y sociales, y de los organismos recaudadores de las ramas profesionales.

\subsubsection{Una gobernanza que sigue siendo dual}

La dualidad de las autoridades públicas responsables entre el Departamento, por una parte, y el seguro de enfermedad, por otra parte, no debería ser cuestionada por la creación de un quinto riesgo. La ley del 21 de julio de 2009 referente a la reforma del hospital y relativa a los pacientes, a la sanidad y a los territorios, conocida como HPST, modificaba únicamente 
las relaciones territoriales de la Caja nacional de solidaridad para la autonomía, que dejaban de ser los servicios desconcentrados del Estado sino las Agencias regionales de sanidad. Este rechazo de elegir entre una política totalmente descentralizada y una rama de la Seguridad social, como durante la creación de la ayuda personalizada de autonomía en 2001, es sin embargo una fuente de complejidad y perjudica una dirección coherente del sector. Durante el debate sobre la ley HPST, ciertos parlamentarios propusieron que se experimentase en ciertos Departamentos la delegación al Consejo general de los créditos de la Caja nacional de solidaridad para la autonomía . Esta vía parece ser fecunda para avanzar de manera progresiva hacia una organización unificada del quinto riesgo.

\section{Conclusiones}

Recordemos que este artículo ha analizado la política de dependencia en Francia, extrayendo las siguientes conclusiones.

En primer lugar, si bien es cierto que las reformas llevadas a cabo desde el año 2000 han sentado las bases de una política de atención de la dependencia, éstas siguen siendo insuficientes. En efecto, estos cambios normativos han desarrollado y estructurado la política de atención de la pérdida de autonomía de diferentes maneras: a) los primeros dispositivos están marcados por las lógicas restrictivas de la ayuda y de la acción social, b) en 2001, se produce la creación de la ayuda personalizada de autonomía, primer dispositivo universal que se convierte rápidamente en una prestación de masas, c) asistimos a un desarrollo planificado de la oferta de servicios desde la canícula del verano de 2003, que privilegia la calidad tratándose de la oferta de centros y de la cantidad en cuanto a la oferta a domicilio, y d) unos gastos directamente destinados a la atención de la dependencia que representan hoy en día aproximadamente el 1 por ciento del PIB. No en vano, estas medidas no permiten hacer frente al incremento de las necesidades estructurales, teniendo en cuenta que: a) las familias asumen unos gastos considerables, b) la política de atención de la dependencia padece de una imagen negativa en la opinión pública, y c) el envejecimiento de la población durante las próximas décadas provocará un incremento de las necesidades.
En segundo lugar, la creación de un quinto riesgo ofrece la oportunidad de realizar unas elecciones en términos de socialización del gasto y de reunir las condiciones de evolución adaptada y eficiente de la oferta de servicios a las personas concernidas. De hecho, unas decisiones estructurales deben permitir fijar el grado de socialización del gasto y sus modalidades: a) diseñando las vías de una mejor atención de las cantidades que deben asumir las personas dependientes y sus familiares, b) enfrentándose a las dificultades de la convergencia con los dispositivos destinados a las personas discapacitadas, c) planteando la cuestión de la toma en consideración de las rentas y del patrimonio por la ayuda personalizada de autonomía, d) y contemplando soluciones para financiar las nuevas necesidades. Simultáneamente, la organización del quinto riesgo deberá favorecer una evolución adecuada y eficiente de la oferta de servicios a las personas concernidas, lo que supone: a) la dirección de la evolución de las estructuras, b) la toma en consideración de las dificultades de contratación del personal, y c) la necesidad de enfrentarse a una gobernanza dual.

Conviene recordar que la atención de la dependencia es el presupuesto menos importante en lo que se refiere al gasto adicional generado por el envejecimiento de la población, lejos por detrás de las pensiones y del gasto sanitario. No obstante, cristaliza todas las preocupaciones sobre la financiación de esta evolución demográfica. Inquietud de las personas concernidas y de sus familias, que temen no poder asumir el elevado coste que puede generar la dependencia en los últimos años de la vida. Preocupación de los poderes públicos, que la explosión de la ayuda personalizada de autonomía ha convertido en muy prudentes y que se enfrentan a la crisis financiera. Concebida como una perspectiva de extensión de una protección social de lógica universal, la institución del quinto riesgo podría finalmente resultar de un difícil arbitraje, conduciendo a un cierto reajuste de la socialización. No en vano, los beneficios de una socialización creciente en términos de empleos creados y de calidad de vida de las personas concernidas y de sus ayudantes no deben ser descuidados, y deben, sin duda, ser integrados de manera óptima en los estudios de impacto de las futuras reformas. 


\section{Referencias bibliográficas}

Baromètre (20 de mayo de 2009). Les Français et le grand âge. TNS Sofres, 20 mai 2009.

Branchu, C. et al, (2009). Etat des lieux relatif à la composition des coûts mis à la charge des résidents des établissements d'hébergement pour personnes âgées dépendantes. Paris: IGAS.

DRESS, Enquêtes EHPA. Etudes et résultats, 379 y 689.

DRESS, (2006). Dépendance des personnes âgées et handicap: les opinions des Français entre 2000 et 2005. Etudes et résultats, 491.

Fédération Française des Sociétés d'Assurance (2008). Les contrats d'assurance dépendance en 2007.

Laroque, P. (1962). Rapport de la commission d'études des problèmes de la vieillesse. Paris: La Documentation française.

Mercier, M. (2005). Le RMI: d'un transfert de gestion à une décentralisation de responsabilité. Rapport d'information fait au nom de l'observatoire de la décentralisation du Sénat.

Sueur, J-P. (2000). L'aide personnalisée à l'autonomie, un Nouveau droit fondé sur le principe d'égalité. Rapport remis au ministre de l'Emploi y de la Solidarité.

Urteaga. E. (2009a). Las políticas de lucha contra la pobreza y la exclusión social en Francia, Zerbitzuan, 45, 7-21.

Urteaga, E. (2009b). Fundamentos y organización de las políticas sociales en Francia. Portularia, $9,(2), 75-95$.

Urteaga, E. (2010a). La territorialización de las políticas sociales. Zerbitzuan, 48, 97-107.

Urteaga, E. (2010b). La política a favor de los discapacitados en Francia, Portularia, 10 (2), 77-89.

Urteaga, E. (2011). Las rentas mínimas en Francia. Papers, 86 (3).

Vasselle, A. (2008). Construire le cinquième risque, le rapport d'étape. Mission commune d'information dépendance du Sénat. 